06

\title{
Модифицированные параметры приема 1-3-композитов на основе сегнетоэлектрических кристаллов
}

\author{
(C) В.Ю. Тополов, А.Н. Исаева
}

Южный федеральный университет, 344090 Ростов-на-Дону, Россия

e-mail: vutopolov@sfedu.ru

Поступило в Редакцию 17 ноября 2020 г.

В окончательной редакции 22 января 2021 r.

Принято к публикации 24 января 2021 г.

Исследована система модифицированных параметров приема волокнистых пьезоактивных композитов „система однонаправленных сегнетоэлектрических кристаллических стержней - полимерная матрица“ со связностью 1-3 в широком интервале объемных концентраций кристаллического компонента. Модифицированные параметры приема важны для оценки эффективности захвата, накопления и преобразования энергии в пьезоэлементе при постоянном механическом напряжении или постоянной деформации. В качестве пьезоэлектрического компонента выступают поляризованные вдоль кристаллографического направления [001] полидоменные кристаллы $(1-x) \mathrm{Pb}\left(\mathrm{Mg}_{1 / 3} \mathrm{Nb}_{2 / 3}\right) \mathrm{O}_{3}-x \mathrm{PbTiO}_{3} \quad(0.28 \leq x \leq 0.33)$ и $\mathrm{Li}_{v}\left(\mathrm{~K}_{1-y} \mathrm{Na}_{y}\right)_{1-v}\left(\mathrm{Nb}_{1-z} \mathrm{Ta}_{z}\right) \mathrm{O}_{3}: \mathrm{Mn}(v=0.06, y=0.1 \ldots 0.3, z=0.07 \ldots 0.17)$. Проведено сравнение параметров, рассчитанных для 1-3-композитов по матричному методу и методу эффективного поля. Проанализирована роль электромеханических свойств кристаллического компонента в формировании модифицированных параметров приема композита. На основе результатов численного моделирования эффективных свойств и связанных с ними модифицированных параметров приема показаны преимущества бессвинцового 1 -3-композита по сравнению с аналогами на основе кристаллов $(1-x) \mathrm{Pb}\left(\mathrm{Mg}_{1 / 3} \mathrm{Nb}_{2 / 3}\right) \mathrm{O}_{3}-x \mathrm{PbTiO}_{3}$.

Ключевые слова: пьезоактивный композит, эффективные электромеханические свойства, параметр приема, фактор анизотропии, свинецсодержащие и бессвинцовые компоненты.

DOI: 10.21883/JTF.2021.06.50867.319-20

\section{Введение}

Пьезоэлектрические материалы представляют академический и практический интерес с точки зрения эффективности преобразования и накопления энергии [15]. Развитие энергосберегающих технологий и создание новых устройств, действие которых основано на пьезоэлектрическом эффекте, требуют знания критериев отбора материалов $[1,2,5,6]$, преобразующих механическую энергию в электрическую и наоборот. Элементы, изготовленные из пьезоэлектрических материалов, могут испытывать действия различных механических, тепловых и электрических полей $[2,4,6,7]$. В связи с этим следует отметить работы [2,3,5-7], где проанализированы параметры приема (ПП), связанные с электромеханическими (т.е. упругими, пьезоэлектрическими и диэлектрическими) свойствами материалов. Наряду с коэффициентами электромеханической связи, используемыми для оценки эффективности преобразования энергии [8], ПП важно учитывать при анализе цикла преобразования энергии для различных мод внешнего воздействия на пьезоэлектрический элемент.

В работе [6] развита концепция модифицированных ПП, применимых в случаях воздействия на пьезоэлектрический элемент механического напряжения $\sigma=$ const или деформации $\xi=$ const. Авторы [6] проанализировали поведение модифицированных ПП пори- стых материалов на основе сегнетопьезокерамик (СПК). Однако до настоящего времени не проводился сравнительный анализ модифицированных ПП монолитных пьезоэлектрических материалов, включая композиты, состоящие из монолитных компонентов. В настоящей работе мы рассматриваем пьезоактивные композиты со связностью 1-3, которые являются важными объектами исследований (см., например, работы $[3,9,10]$ ). Это обусловлено относительно простой структурой $1-3$-композитов $[3,11]$, хорошо апробированными методами получения этих материалов [9-11] и особенностями их эффективных свойств [3,9-13]. Отдельно выделим работы по бессвинцовым 1-3-композитам [9,10,12], их эффективным свойствам и применениям.

На протяжении последних десятилетий сформировались несколько подходов к определению эффективных свойств композитов [14-17]. Основные принципы вычислительной механики композитов и стадии прогнозирования их эффективных свойств изложены в работе [15]. Наряду с асимптотическими методами осреднения свойств [14] находят применение методы вириального разложения, самосогласования (схемы методов эффективной среды и эффективного поля) [16-20], метод функций Грина [21,22], метод асимптотической гомогенизации [23], матричный метод [3,24] и метод конечных элементов [25-27]. Большой практический интерес представляют методы определения эффективных 
свойств пьезо- и пироактивных композитов $[15,16]$ с различной связностью. Важные результаты по эффективным свойствам пьезоактивных и родственных композитов получены авторами работ [22,28], где рассмотрены стохастические и квазипериодические структуры, примеры многокомпонентных структур и развиты методы прогнозирования свойств для подобных структур. Анализ литературных данных по эффективным свойствам пьезоактивных композитов показывает, что в большинстве работ исследованы материалы типа „СПК-полимер“.

Примеры эффективных электромеханических свойств 1-3-композитов на основе кристаллов релаксоровсегнетоэлектриков $(1-x) \mathrm{Pb}\left(\mathrm{Mg}_{1 / 3} \mathrm{Nb}_{2 / 3}\right) \mathrm{O}_{3}-x \mathrm{PbTiO}_{3}$ $(\mathrm{PMN}-x \mathrm{PT}) \quad$ с высокой пьезоактивностью впервые описаны в работе [29]. Сравнения эффективных свойств, определенных для 1-3-композитов с использованием различных методов, проведены в работах [25] (матричный метод и метод конечных элементов) и [26] (метод эффективного поля и метод конечных элементов).

Цель настоящей работы - анализ концентрационных зависимостей системы модифицированных ПП 1-3-композитов на основе сегнетоэлектрических кристаллов и сравнение значений ПП свинецсодержащих и бессвинцовых композитов.

\section{1. Модель пьезоактивного 1-3-композита, его эффективные свойства и параметры приема}

\section{1. Структура и эффективные свойства 1-3-композита „кристалл-полимер“}

Исследуемый 1-3-композит представляет собой систему протяженных параллельных кристаллических стержней, окруженных непрерывной полимерной матрицей (рис. 1). На вставке 1 рис. 1 показаны ориентации векторов спонтанной поляризации $\mathbf{P}_{s, f}$ отдельных типов доменов $(f=1,2,3,4)$, стрелкой справа показано направление внешнего электрического поля $\mathbf{E}$, приводящего к данной ориентации доменов („domain-engineered

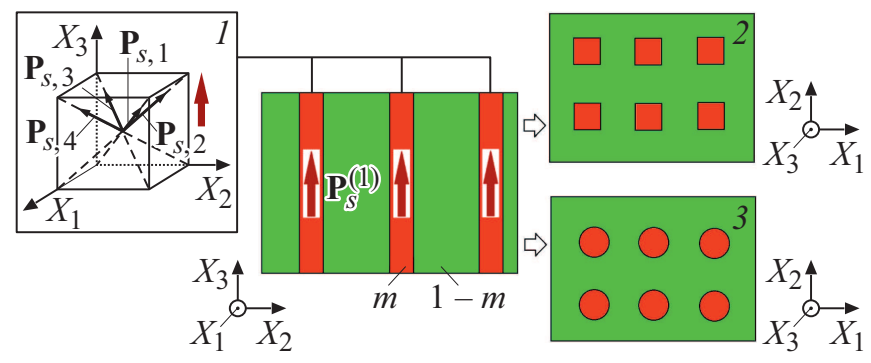

Рис. 1. Схематическое изображение сечения 1-3-композита „сегнетоэлектрический кристалл-полимер“плоскостью $\left(X_{2} O X_{3}\right) .\left(X_{1} X_{2} X_{3}\right)$-прямоугольная система координат, $\mathbf{P}_{s}^{(1)}-$ вектор спонтанной поляризации кристаллического стержня, $m$ и $1-m-$ объемные концентрации кристаллического и полимерного компонента соответственно. crystal“", см. например, работу [30]). Каждый кристаллический стержень характеризуется вектором спонтанной поляризации $\mathbf{P}_{s}^{(1)}$, который сонаправлен с $\left(O X_{3}\right)$. Сечения плоскостью $\left(X_{1} O X_{2}\right) 1-3$-композитов со стержнями в форме прямоугольного параллелепипеда с квадратным основанием и в форме кругового цилиндра изображены соответственно на вставках 2 и 3 рис. 1. Главные кристаллографические оси каждого стержня $X, Y, Z$ ориентированы вдоль направлений кубической элементарной ячейки следующим образом: ось $X$ параллельна направлению [100] и оси координат $\left(O X_{1}\right)$, ось $Y$ параллельна [010] и $\left(O X_{2}\right)$, ось $Z$ параллельна [001] и $\left(O X_{3}\right)$. При этом ось координат $\left(O X_{3}\right)$ является осью поляризации композита в целом. Предполагается, что центры оснований всех стержней в плоскости $\left(X_{1} O X_{2}\right)$ формируют простую квадратную сетку (см. вставки 2 и 3 на рис. 1).

Эффективные электромеханические свойства 1-3-композита со стержнями в форме прямоугольного параллелепипеда определяются с использованием матричного метода [3,24], а свойства 1-3-композита с цилиндрическими стержнями определяются по методу эффективного поля $[3,16,31]$. В обоих указанных методах учитывается электромеханическое взаимодействие между пьезоэлектрическими стержнями. При этом в матричном методе мы используем электромеханические константы компонентов из определяющих соотношений электроупругой среды $[8,27]$

$$
\xi_{p}=s_{p q}^{E} \sigma_{q}+d_{f p} E_{f} ; D_{k}=d_{k l} \sigma_{l}+\varepsilon_{k r}^{\sigma} E_{r},
$$

a в методе эффективного поля-константы компонентов из определяющих соотношений $[8,27]$ $\sigma_{p}=c_{p q}^{E} \xi_{q}-e_{f p} E_{f} ; \quad D_{k}=e_{k l} \xi_{l}+\varepsilon_{k r}^{\xi} E_{r}, \quad$ где $\quad \xi_{p}$, $\sigma_{q}, E_{f}, D_{k}-$ соответственно механическая деформация, механическое напряжение, напряженность электрического поля, электрическое смещение. Упругие, пьезоэлектрические и диэлектрические константы $\left(s_{p q}^{E}\right.$, $d_{f p}$ и $\varepsilon_{k r}^{\sigma}$ в матричном методе или $c_{p q}^{E}, e_{f p}$ и $\varepsilon_{k r}^{\xi}$ в методе эффективного поля) образуют полные наборы электромеханических констант [8], которые представлены в двухиндексной (т.е. матричной) форме. Выбор матричного метода и метода эффективного поля связан с различными формами пьезоэлектрических стержней 1-3-композитов (см. вставки 2 и 3 на рис. 1), а также с важностью сопоставления результатов, получаемых при различных процедурах усреднения с использованием различных полных наборов электромеханических констант компонентов. В обоих методах [3,16,24,31] внешние механические или электрические поля, действующие на композитный образец, считаются слабыми, а возникающие во внешнем поле Е электрострикцонные деформации - пренебрежимо малыми по сравнению с пьезодеформациями (слагаемыми типа $d_{f p} E_{f}$ в $\left.(1)\right)$.

Матрица эффективных свойств, определяемых в рамках матричного метода для рассматриваемого 
1-3-композита (рис. 1), имеет вид

$$
\begin{aligned}
& \left\|\mathbf{C}_{\text {matr }}^{*}\right\|=\left[\left\|\mathbf{C}^{(1)}\right\| \cdot\|\mathbf{M}\| m+\left\|\mathbf{C}^{(2)}\right\|(1-m)\right] \\
& \times[\|\mathbf{M}\| m+\|\mathbf{I}\|(1-m)]^{-1},
\end{aligned}
$$

где $\left\|\mathbf{C}^{(1)}\right\|, \quad\left\|\mathbf{C}^{(2)}\right\|-\quad$ матрицы электромеханических свойств кристаллического и полимерного компонентов соответственно, $\|\mathbf{M}\|$ - матрица, содержащая компоненты обобщенных „тензоров концентраций“ (по терминологии [14]), $\|\mathbf{I}\|$ - единичная матрица, $m$ - объемная концентрация кристаллического компонента в композите, „-1“ в правой части используется для обозначения матрицы, обратной матрице $\|\mathbf{M}\| m+\|\mathbf{I}\|(1-m)$. Матрицы $\left\|\mathbf{C}^{(1)}\right\|$ и $\left\|\mathbf{C}^{(2)}\right\|$ из (2) представляются в виде блочной матрицы

$$
\left\|\mathbf{C}^{(n)}\right\|=\left(\begin{array}{cc}
\left\|S^{(n), E}\right\| & \left\|d^{(n)}\right\|^{t} \\
\left\|d^{(n)}\right\| & \left\|\mathcal{E}^{(n), \sigma}\right\|
\end{array}\right),
$$

и матрица $\left\|\mathbf{C}_{\text {matr }}^{*}\right\|$ из (2) имеет структуру, аналогичную $\left.\| \mathbf{C}^{(n}\right) \|$ из (3). Размерность всех матриц в формулах (2) и (3) $-9 \times 9$. В формуле (3) $\left\|\mathbf{s}^{(n), E}\right\|-$ матрица упругих податливостей $n$-го компонента при $E=$ const (размерность матрицы $6 \times 6$ ), $\left\|\mathbf{d}^{(n)}\right\|-$ матрица пьезомодулей $n$-го компонента (размерность матрицы $3 \times 6$ ), $\left\|\varepsilon^{(n), \sigma}\right\|-$ матрица диэлектрических проницаемостей $n$-го компонента при $\sigma=$ const (размерность матрицы $3 \times 3), n=1$ соответствует кристаллу, $n=2$ - полимеру, верхний индекс , $t^{\text {“6 } о б о з н а ч а е т ~ т р а н с п о н и р о в а н и е ~}$ матрицы. Элементы матриц в правой части формулы (3) формируют полные наборы электромеханических констант по аналогии с константами из правых частей соотношений (1).

В модели композита (рис. 1) кристаллические стержни считаются бесконечно длинными вдоль оси $\left(O X_{3}\right)$, а их боковые грани идеально плоскими. Матрица $\|\mathbf{M}\|$ из (2) формируется [27] с учетом свойств кристалла и полимера и непрерывности:

а) компонент механических напряжений $\sigma_{11}, \sigma_{12}, \sigma_{13}$, деформаций $\xi_{22}, \xi_{23}, \xi_{33}$ (в одноиндексной форме записи это $\left.\sigma_{1}, \sigma_{6}, \sigma_{5}, \xi_{2}, \xi_{4}, \xi_{3}\right)$, напряженности электрического поля $E_{2}, E_{3}$ и электрического смещения $D_{1}$ на плоских границах раздела „стержень - матрица“, перпендикулярных оси $\left(O X_{1}\right)$;

б) компонент механических напряжений $\sigma_{12}, \sigma_{22}, \sigma_{23}$, деформаций $\xi_{11}, \xi_{13}, \xi_{33}$ (в одноиндексной форме это $\sigma_{6}$, $\left.\sigma_{2}, \sigma_{4}, \xi_{1}, \xi_{5}, \xi_{3}\right)$, напряженности электрического поля $E_{1}$, $E_{3}$ и электрического смещения $D_{2}$ на плоских границах раздела „стержень-матрица“, перпендикулярных оси $\left(\mathrm{OX}_{2}\right)$.

В частности, для боковых граней стержней, перпендикулярных оси $\left(O X_{1}\right)$, матрица $\|\mid \mathbf{M}\|$ в соответствии с представлениями работ [14,27] имеет общий вид

$$
\begin{aligned}
& \|\mathbf{M}\|=\left\|\mathbf{p}_{1}\right\|^{-1}\left\|\mathbf{p}_{2}\right\|, \text { где } \\
& \left\|p_{n}\right\|= \\
& =\left(\begin{array}{cccccccccc}
1 & 0 & 0 & 0 & 0 & 0 & 0 & 0 & 0 \\
s_{12}^{(n), E} & s_{22}^{(n), E} & s_{23}^{(n), E} & s_{24}^{(n), E} & s_{25}^{(n), E} & s_{26}^{(n), E} & d_{12}^{(n)} & d_{22}^{(n)} & d_{32}^{(n)} \\
s_{13}^{(n), E} & s_{23}^{(n), E} & s_{33}^{(n), E} & s_{34}^{(n), E} & s_{35}^{(n), E} & s_{36}^{(n), E} & d_{13}^{(n)} & d_{23}^{(n)} & d_{33}^{(n)} \\
s_{14}^{(n), E} & s_{24}^{(n), E} & s_{34}^{(n), E} & s_{44}^{(n), E} & s_{45}^{(n), E} & s_{46}^{(n), E} & d_{14}^{(n)} & d_{24}^{(n)} & d_{34}^{(n)} \\
0 & 0 & 0 & 0 & 1 & 0 & 0 & 0 & 0 \\
0 & 0 & 0 & 0 & 0 & 1 & 0 & 0 & 0 \\
d_{11}^{(n)} & d_{12}^{(n)} & d_{13}^{(n)} & d_{14}^{(n)} & d_{15}^{(n)} & d_{16}^{(n)} & \varepsilon_{11}^{(n), \sigma} & \varepsilon_{12}^{(n), \sigma} & \varepsilon_{13}^{(n), \sigma} \\
0 & 0 & 0 & 0 & 0 & 0 & 0 & 1 & 0 \\
0 & 0 & 0 & 0 & 0 & 0 & 0 & 0 & 1
\end{array}\right)
\end{aligned}
$$

- вспомогательная матрица, которая содержит электромеханические константы кристалла $(n=1)$ или полимеpa $(n=2)$. Матрицы $\left\|\mathbf{p}_{1}\right\|$ и $\left\|\mathbf{p}_{2}\right\|$ являются связующими звеньями в условиях непрерывности компонент механических и электрических полей на плоской границе раздела. Эти условия с учетом формул (1) и свойств кристалла и полимера принимают компактную форму

$$
\left\|p_{1}\right\|\left(\begin{array}{l}
\left\|\sigma^{(1)}\right\| \\
\left\|\mathbf{E}^{(1)}\right\|
\end{array}\right)=\left\|p_{2}\right\|\left(\begin{array}{c}
\left\|\boldsymbol{\sigma}^{(2)}\right\| \\
\left\|\mathbf{E}^{(2)}\right\|
\end{array}\right),
$$

где $\left\|\boldsymbol{\sigma}^{(n)}\right\|$ - матрица-столбец, содержащая компоненты $\sigma_{1}, \sigma_{2}, \ldots, \sigma_{6},\left\|\mathbf{E}^{(n)}\right\|-$ матрица-столбец, содержащая компоненты $E_{1}, E_{2}, E_{3} ; n=1 ; 2$. Строки матрицы $\left\|\mathbf{p}_{n}\right\|$ из (4), которые содержат электромеханические константы, имеют тот же вид, что и соответствующие строки $\left.\| \mathbf{C}^{(n)}\right) \|$ из (3).

В матричном методе сделано предположение об однородности механических напряжений $\sigma_{i}$ и электрических полей $E_{k}$ в компонентах1-3-композита, и именно $\sigma_{i}$ и $E_{k}$ присутствуют в правых частях выражений (1). Нами не учитываются переходные области между стержнями и полимерной средой вследствие незначительной диффузии [10-12] между разнородными неорганическими и органическими компонентами композита. Для сравнения укажем, что расчеты полного набора эффективных электромеханических констант полидоменного сегнетоэлектрического кристалла (аналога слоистого композита со связностью 2-2) впервые проведены Туриком [32] в приближении однородных полей $\sigma_{i}$ и $E_{k}$ в соседних $90^{\circ}$-ных доменах и при бесконечно тонких плоских границах между ними. В работе [32] записаны соотношения вида (1) с учетом ориентаций кристаллографических осей каждого типа $90^{\circ}$-ных доменов и с учетом симметрии $4 m m$ отдельного домена. По аналогии с представлениями работ [32,33], мы считаем постоянными свойства стержней и окружающей матрицы вдоль боковых плоских границ раздела (рис. 1), а эти границы считаем достаточно 
тонкими, слабо влияющими на отношение объемных концентраций компонентов $m /(1-m)$ в композитном образце. На данных границах раздела внутри композита не учитываются „шероховатости“, вызванные различными ориентациями не $180^{\circ}$-ных доменов в стержнях (вставка 1 на рис. 1), анизотропными деформациями каждого типа доменов во внешних полях и возможным присутствием межфазных границ в гетерофазных составах [8] вблизи морфотропной фазовой границы. Полный набор электромеханических констант стержня характеризует его как эффективную (гомогенизированную) среду с учетом различных типов доменов, их объемных концентраций, ориентаций и пространственного распределения по кристаллу [30], а также с учетом гетерофазных областей в составах вблизи морфотропной фазовой границы [8]. Эффективные свойства, характеризующиеся матрицей $\left\|\mathbf{C}_{\text {matr }}^{*}\right\|$ из (2), удовлетворяют условиям длинноволнового приближения [34], т.е. когда длина волны внешнего поля (электрического или акустического) значительно больше длины отдельного стержня композита. В этом случае, например, пренебрегают различиями между значениями напряженности внешнего периодического поля $E$ из-за пренебрежимо малой разности фаз в различных точках образца.

Среди преимуществ матричного метода можно отметить компактность основных формул (2) и (3), а также использование в матрицах $\left\|\mathbf{C}^{(n)}\right\|,\|\mathbf{M}\|$ из (2), (3) и $\left\|\mathbf{p}_{n}\right\|$ из (4) электромеханических констант, кратных $10^{-12}$ в единицах СИ. Например, элементы $\left\|\mathbf{s}^{(n), E}\right\|$ компонентов задаются в $10^{-12} \mathrm{~Pa}^{-1}$, а элементы $\left\|\mathbf{d}^{(n)}\right\|-$ в $10^{-12} \mathrm{C} / \mathrm{N}$. В матричном методе нет ограничений, налагаемых на симметрию свойств компонентов, а изменение объемной концентрации стержней $m$ вследствие планарной микрогеометрии композита возможно в интервале $0 \leq m \leq 1$ [24,25,27]. Примеры согласия между концентрационными зависимостями эффективных пьезокоэффициентов, рассчитанных для 1-3-композитов „сегнетоэлектрический кристалл- сегнетоэлектрический полимер“ по матричному методу и методу конечных элементов, приведены в работе [25].

До разработки матричного метода определение эффективных свойств и гидростатических параметров 1-3-композита „СПК - пьезопассивный полимер“ с системой стержней в форме прямоугольного параллелепипеда проводилось [33] при записи определяющих соотношений (1) и условий непрерывности в переменных $\sigma_{i}$ и $E_{k}$. Однако в работе [33] не рассмотрен полный набор электромеханических констант 1-3-композита даже для фиксированной объемной концентраци $m$.

Определяемую по методу эффективного поля матрицу эффективных свойств 1-3-композита с цилиндрическими стержнями $[3,25]$ можно представить как

$$
\begin{aligned}
& \left\|\mathbf{C}_{E F M}^{*}\right\|=\left\|\mathbf{K}^{(2)}\right\|+m\left(\left\|\mathbf{K}^{(1)}\right\|-\left\|\mathbf{K}^{(2)}\right\|\right)[\|\mathbf{I}\| \\
& \left.+(1-m)\|\mathbf{S}\| \cdot\left\|\mathbf{K}^{(2)}\right\|^{-1}\left(\left\|\mathbf{K}^{(1)}\right\|-\left\|\mathbf{K}^{(2)}\right\|\right)\right]^{-1} .
\end{aligned}
$$

В формуле (5) мы используем „-1“ для обозначения матрицы, обратной данной. При этом структура блочной матрицы

$$
\left\|\mathbf{C}_{E F M}^{*}\right\|=\left(\begin{array}{cc}
\left\|c^{* E}\right\| & \left\|e^{*}\right\|^{t} \\
\left\|e^{*}\right\| & -\left\|\varepsilon^{* o}\right\|
\end{array}\right)
$$

отличается от представленной в (3). Матрицы в правой части формулы (6) характеризуют модули упругости при $E=\operatorname{const}\left(\left\|\mathbf{c}^{* E}\right\|\right.$, размерность $\left.6 \times 6\right)$, пьезокоэффициенты $\left(\left\|\mathbf{e}^{*}\right\|\right.$, размерность $\left.3 \times 6\right)$ и диэлектрические проницаемости при $\xi=$ const $\left(\left\|\varepsilon^{* \xi}\right\|\right.$, размерность $\left.3 \times 3\right)$. Формула (5) записана с учетом предположения об однородности полей деформаций и напряженностей электрического поля в кристаллическом стержне. В этом случае не учитываются особенности распределения деформаций и электрических полей внутри стержня при переходе от одного домена к другому (вставка 1 на рис. 1), и переходный слой между стержнем и матрицей предполагается бесконечно тонким. В формуле (5) матрицы $\left\|\mathbf{K}^{(1)}\right\|$ и $\left\|\mathbf{K}^{(2)}\right\|$ характеризуют свойства кристалла и полимера соответственно, а структура матриц $\left\|\mathbf{K}^{(n)}\right\|$ аналогична структуре $\left\|\mathbf{C}_{E F M}^{*}\right\|$ из (6). Матрица $\|\mathbf{S}\|$ из (6) содержит компоненты тензора Эшелби $[3,35]$, зависящего от свойств полимерной матрицы и от формы границы раздела компонентов (т.е. от боковой поверхности цилиндра в рассматриваемом случае). Добавим, что в методе эффективного поля [3,31] среда, окружающая стержни, может быть либо изотропной, либо трансверсально изотропной. При заполнении композита протяженными цилиндрическими стержнями с регулярным расположением (вставка 3 на рис. 1) объемная концентрация стержней ограничена сверху значением $m_{u p}=\pi / 4$. Сравнение эффективных свойств 1-3-композитов „СПК - пьезопассивный полимер“ которые определены по методам эффективного поля и конечных элементов в работе [26], указывает на хорошее согласие между расчетными результатами.

\section{2. Модифицированные параметры приема композита}

При исследовании пьезоактивных 1-3-композитов вызывает интерес немонотонное концентрационное поведение квадратов ПП [3,27,36,37]

$$
\left(Q_{3 j}^{*}\right)^{2}=d_{3 j}^{*} g_{3 j}^{*} \text { и }\left(Q_{h}^{*}\right)^{2}=d_{h}^{*} g_{h}^{*},
$$

которые используются для оценки отношения „сигнал-шум“. В формулах (7) $g_{3 j}^{*}-$ пьезокоэффициенты, $d_{h}^{*}$ и $g_{h}^{*}$ - гидростатические пьезокоэффициенты, индекс $j=1$ или 2 соответствует поперечному пьезоэффекту, a $j=3$ - продольному пьезоэффекту. Следуя концепции [5-7,38], мы рассматриваем четыре вида модифицированных ПП, связанных с продольным и поперечным пьезооткликами композита ${ }^{1}$ ):

\footnotetext{
${ }^{1}$ Учитывая слабый сдвиговый пьезоэффект по сравнению с продольным или поперечным пьезоэффектом в различных 1-3-композитах на основе СПК или сегнетоэлектрических кристаллов [27], мы не рассматриваем ПП $\lambda_{15, m}^{*}, L_{15}^{*}$ и т. П.
} 
Таблица 1. Упругие податливости $s_{a b}^{E}$, пьезомодули $d_{i j}$ и диэлектрические проницаемости $\varepsilon_{p p}^{\sigma}$ компонентов исследуемых композитов

\begin{tabular}{c|c|c|c|c|c|c|c|c|c|c|c}
\hline & $\begin{array}{c}s_{11}^{E}, \\
10^{-12} \\
\mathrm{~Pa}^{-1}\end{array}$ & $\begin{array}{c}s_{12}^{E}, \\
10^{-12} \\
\mathrm{~Pa}^{-1}\end{array}$ & $\begin{array}{c}s_{13}^{E}, \\
10^{-12} \\
\mathrm{~Pa}^{-1}\end{array}$ & $\begin{array}{c}s_{33}^{E}, \\
10^{-12} \\
\mathrm{~Pa}^{-1}\end{array}$ & $\begin{array}{c}s_{44}^{E}, \\
10^{-12} \\
\mathrm{~Pa}^{-1}\end{array}$ & $\begin{array}{c}s_{66}^{E}, \\
10^{-12} \\
\mathrm{~Pa}^{-1}\end{array}$ & $\begin{array}{c}d_{31}, \\
\mathrm{pC} / \mathrm{N}\end{array}$ & $\begin{array}{c}d_{33}, \\
\mathrm{pC} / \mathrm{N}\end{array}$ & $\begin{array}{c}d_{15}, \\
\mathrm{pC} / \mathrm{N}\end{array}$ & $\begin{array}{c}\varepsilon_{11}^{\sigma} / \varepsilon_{0} \\
\varepsilon_{33} / \varepsilon_{0}\end{array}$ \\
\hline PMN-0.33PT [30] & 69.0 & -11.1 & -55.7 & 119.6 & 14.5 & 15.2 & -1330 & 2820 & 146 & 1600 & 8200 \\
PMN-0.30PT [39] & 52.0 & -18.9 & -31.1 & 67.7 & 14.0 & 15.2 & -921 & 1981 & 190 & 3600 & 7800 \\
PMN-0.29PT [40] & 52.1 & -24.6 & -26.4 & 59.9 & 16.0 & 28.3 & -699 & 1540 & 164 & 1560 & 5400 \\
PMN-0.28PT [41] & 44.57 & -28.91 & -13.91 & 34.38 & 15.22 & 16.34 & -569 & 1182 & 122 & 1672 & 5479 \\
KNNTL-Mn [42] & 33.4 & -7.36 & -25.8 & 57.7 & 12.8 & 13.5 & -260 & 545 & 66 & 400 & 650 \\
Полиуретан [43] & 405 & -151 & -151 & 405 & 1110 & 1110 & 0 & 0 & 0 & 3.5 & 3.5
\end{tabular}

1. Максимальный коэффициент передачи, т. е. наибольшее отношение выходной электрической энергии образца ко входной механической энергии, или наибольшее отношение выходной механической энергии образца ко входной электрической энергии

$$
\lambda_{3 j, m}^{*}=\left[\left(k_{3 j}^{*}\right)^{-1}-\left(\left(k_{3 j}^{*}\right)^{-2}-1\right)^{1 / 2}\right]^{2} .
$$

2. Отношение максимальной выходной электрической энергии образца к максимальной электрической энергии, накопленной в нем, равно

$$
L_{3 j}^{*}=\lambda_{3 j, m}^{*} /\left(k_{3 j}^{*}\right)^{2} .
$$

3. ПП, связанный с воздействием на образец однородного механического напряжения, -

$$
F_{3 j}^{* \sigma}=L_{3 j}^{*}\left(Q_{3 j}^{*}\right)^{2} .
$$

4. ПП, связанный с воздействием на образец однородной механической деформации, -

$$
F_{3 j}^{* \xi}=F_{3 j}^{* \sigma} /\left(s_{j j}^{* E} s_{j j}^{* D}\right) .
$$

В формуле (8) $k_{3 j}^{*}$ - коэффициент электромеханической связи композита, причем $k_{31}^{*}$ или $k_{32}^{*}$ берется по модулю. В формуле (11) $s_{j j}^{* D}-$ упругая податливость при $D=$ const.

\section{3. Компоненты композитов}

Для анализа концентрационных зависимостей модифицированных ПП из (8)-(11) нами использованы измеренные при комнатной температуре [30,39-43] константы компонентов (табл. 1). Пьезоэлектрическими компонентами композитов являются поляризованные вдоль направления [001] кристаллы РMN-xPT при $x=$ $=0.28 \ldots 0.33$ и $\left[\mathrm{Li}_{v}\left(\mathrm{~K}_{1-y} \mathrm{Na}_{y}\right)_{1-v}\right]\left(\mathrm{Nb}_{1-z} \mathrm{Ta}_{z}\right) \mathrm{O}_{3}: \mathrm{Mn}$ (KNNTL-Mn) при $\quad v=0.06, \quad y=0.1-0.3$, $z=0.07-0.17$. Данные кристаллы со структурой типа перовскита и с доменной структурой, показанной схематически на вставке 1 рис. 1 , характеризуются макроскопической симметрией 4mm [30,39-42]. Химический состав кристалла PMN-0.33PT, обладающего наибольшей пьезоактивностью среди перечисленных в табл. 1 соединений, соответствует морфотропной фазовой границе [44]. Бессвинцовый кристалл KNNTL-Mn обладает пьезомодулями $d_{3 j}$, превышающими аналогичные значения многих перовскитовых свинецсодержащих СПК [8,27]. Вторым компонентом исследуемых композитов является полиуретан $[3,43]$.

В табл. 1 представлены электромеханические константы, используемые для формирования $\left\|\mathbf{C}^{(n)}\right\|$ в матричном методе (формулы (1)-(3)). Для расчетов по методу эффективного поля используются константы из матриц $\left\|\mathbf{c}^{(n), E}\right\|, \quad\left\|\mathbf{e}^{(n)}\right\|$ и $\left\|\boldsymbol{\varepsilon}^{(n), \xi}\right\|$. Связи между различными полными наборами электромеханических констант пьезоэлектрической среды описываются с помощью формул [8,27].

\section{2. Концентрационные зависимости эффективных пьезоэлектрических свойств и модифицированных параметров приема 1-3-композитов}

Примеры концентрационных зависимостей эффективных пьезомодулей $d_{3 j}^{*}(m)$ композитов и их модифицированных ПП из формул (8)-(11) графически представлены на рис. 2. Данные зависимости рассчитаны в рамках матричного метода. Рассчитанные по методу эффективного поля концентрационные зависимости аналогичных параметров используются нами для сравнения, см. разд. 3.

Конфигурация кривых пьезомодулей $d_{3 j}^{*}(m)$ (рис. 2,a) обусловлена не только микрогеометрией 1-3-композита (рис. 1), но и модулем разности $\left|s_{a b}^{(1), E}-s_{a b}^{(2), E}\right|$, связанным с различием упругих свойств кристалла и полимера. Благодаря бо́льшей разности $\left|s_{a b}^{(1), E}-s_{a b}^{(2), E}\right|$ пьезомодули $d_{3 j}^{*}(m)$ достигают насыщения при меньшей объемной концентрации $m$ (кривые 3,4 на рис. 2,a). Анализ зависимостей $d_{33}^{*}(m)$ композитов на основе PMN- $x$ PT показывает, что при $m=$ const наибольшее значение $d_{33}^{*}$ соответствует композиту с $x=0.33$, далее следуют $d_{33}^{*}$ композитов с $x=0.30,0.29,0.28$ и $d_{33}^{*}$ композита на ос- 

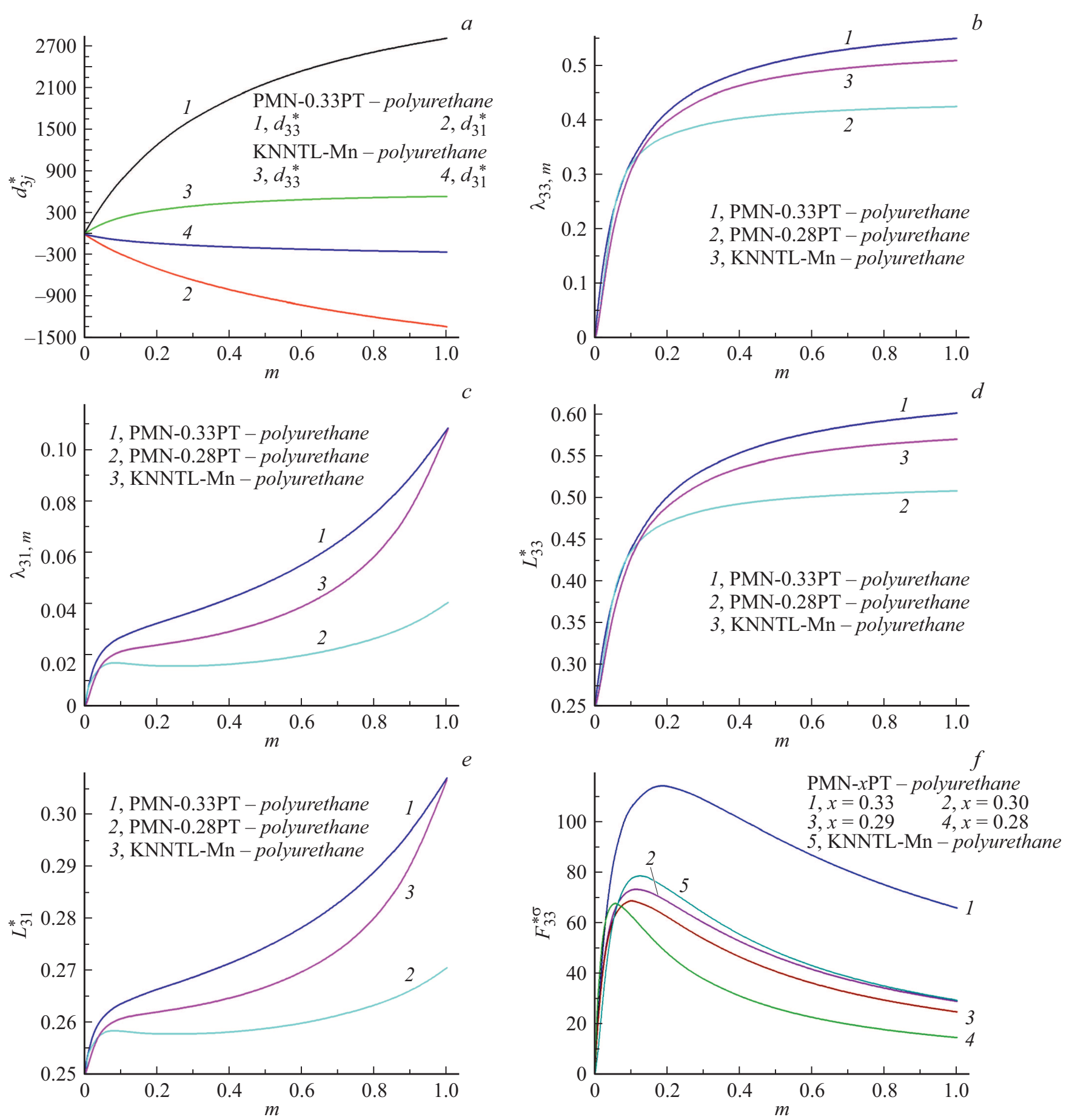

Рис. 2. Концентрационные зависимости эффективных пьезомодулей $d_{3 j}^{*}\left(a-\right.$ в рC/N) и модифицированных ПП $\lambda_{33, m}^{*}(b), \lambda_{31, m}^{*}(c)$, $L_{33}^{*}(d), L_{31}^{*}(e), F_{33}^{* \sigma}\left(f-\right.$ в $\left.10^{-12} \mathrm{~Pa}^{-1}\right), F_{31}^{* \sigma}\left(g-\right.$ в $\left.10^{-12} \mathrm{~Pa}^{-1}\right), F_{33}^{* \xi}\left(h-\right.$ в $\left.10^{10} \mathrm{~Pa}\right), F_{31}^{* \xi}\left(i-\right.$ в $10^{10}$ Ра) $1-3$-композитов с кристаллическими стержнями в форме прямоугольного параллелепипеда.

нове KNNTL-Mn. Данная последовательность согласуется с последовательностью значений пьезомодулей $d_{33}$ кристаллов (табл. 1).

Несмотря на значительные различия между пьезомодулями $d_{3 j}^{*}$ композитов на основе PMN-0.33PT и KNNTL-Mn (рис. 2,a), значения ПП $\lambda_{3 j, m}^{*}$ композита на основе KNNTL-Mn мало отличаются от $\lambda_{3 j, m}^{*}$ композита на основе PMN-0.33PT (кривые 2 и 3 на рис. 2, $b, c$ ). Малые различия наблюдаются также при сравнении ПП $L_{3 j}^{*}$ двух данных композитов (рис. $2, d, e$ ). Не показанные на рис. $2, b-e$ кривые $\lambda_{3 j, m}^{*}(m)$ и $L_{3 j}^{*}(m)$, относящиеся к композитам на основе $\mathrm{PMN}-x \mathrm{PT}$ с $x=0.30,0.29$, могут быть построены в узкой области между кривыми 1 и 2. Отметим большую анизотропию $\lambda_{33, m}^{*} / \lambda_{31, m}^{*}$ в ши- 

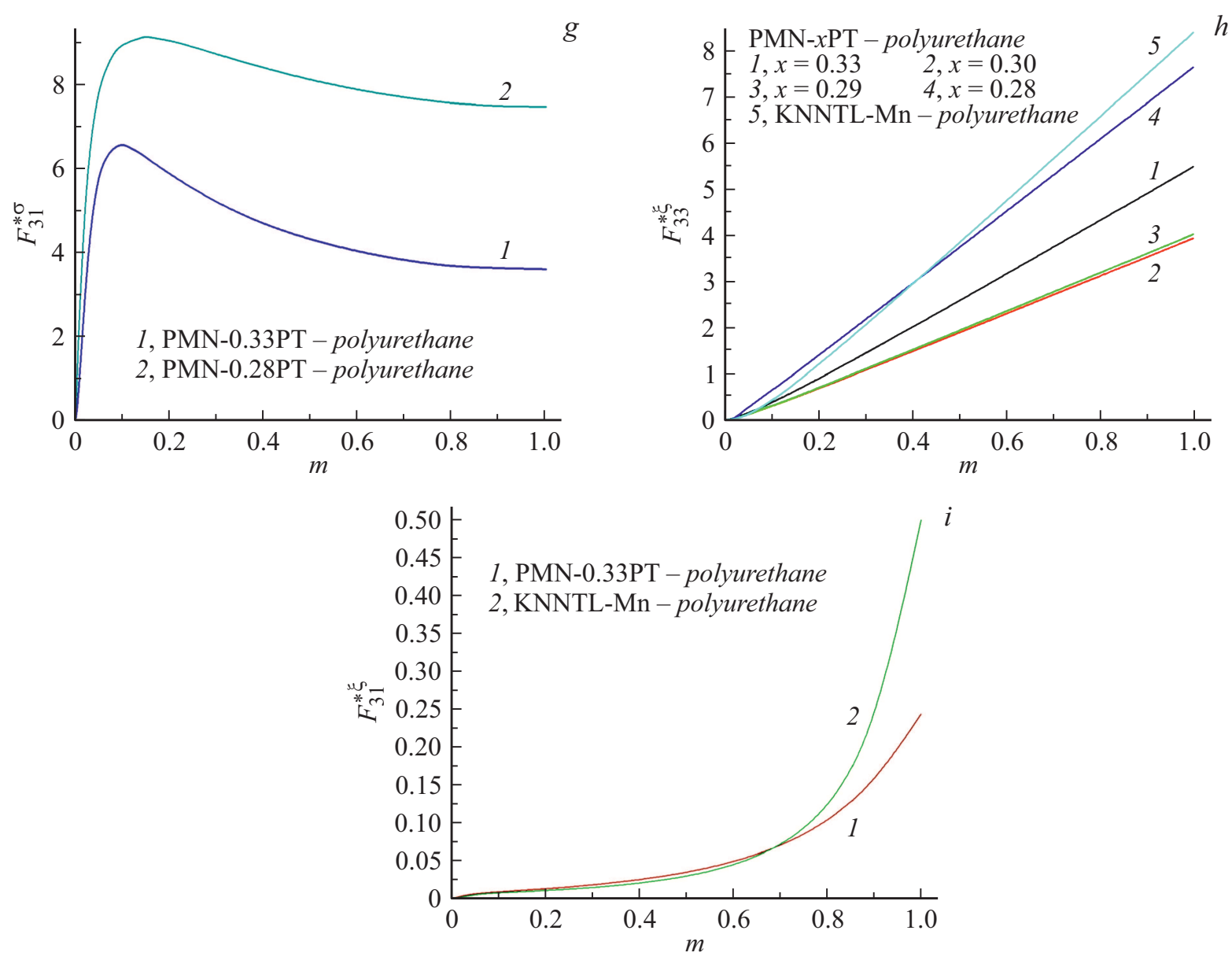

Рис. 2 (продолжение).

роком интервале $m$ (рис. $2, b, c$ ) независимо от состава кристалла композита. Этот эффект связан с микрогеометрией 1-3-композита (рис. 1) и анизотропией его упругих и пьезоэлектрических свойств.

Достаточно большие значения $\lambda_{33, m}^{*}$ и $L_{33}^{*}$ композита на основе KNNTL-Mn (кривые 3 на рис. 2, $b, d$ ) связаны с большим коэффициентом электромеханической связи кристалла $k_{33}^{(1)}$. Примечательно, что кристалл KNNTL-Mn характеризуется значением $k_{33}^{(1)}=0.95$ [42], практически равным $k_{33}^{(1)}$ кристалла PMN-0.33PT [30]. $\mathrm{У}$ исследованных композитов $k_{33}^{*}(m)$ является монотонной зависимостью независимо от кристаллического компонента, что согласуется с результатами [27] по различным пьезоактивным 1-3-композитам.

Зависимости $F_{33}^{* \sigma}(m)$ (рис. $\left.2, f\right)$ показывают, что композит на основе KNNTL-Mn имеет преимущества по сравнению $\mathrm{c}$ композитами на основе $\mathrm{PMN}-x \mathrm{PT} \mathrm{c}$ $x \leq 0.30$. Отметим, что $\max F_{33}^{* \sigma}$ композита на основе KNNTL-Mn достигается при объемной концентрации $m=0.125$ (кривая 5 на рис. $2, f$ ), что облегчает получение 1-3-композитов [11]. Учитывая формулы (7), (10) и пропорциональность $F_{33}^{* \sigma} \sim L_{33}^{*} ; F_{33}^{* \sigma} \sim\left(Q_{33}^{*}\right)^{2} \sim\left(g_{33}^{*}\right)^{2}$, можно утверждать следующее. Большие значения $F_{33}^{* \sigma}$ композита на основе KNNTL-Mn связаны не только с большим $k_{33}^{(1)}$, но и с большим пьезокоэффициентом $g_{33}^{(1)}=94.7 \mathrm{mV} \cdot \mathrm{m} / \mathrm{N}$ [42], что обусловлено низкой диэлектрической проницаемостью $\varepsilon_{33}^{(1), \sigma}$ KNNTL-Mn по сравнению с $\varepsilon_{33}^{(1), \sigma} \mathrm{PMN}-0.33 \mathrm{PT}$ (табл. 1 и работы $[30,42])$.

Фактор анизотропии ПП $F_{33}^{* \sigma} / F_{31}^{* \sigma}$ (ср. рис. $2, f$ и $g$ ) при $m=$ const выше, чем $\lambda_{33, m}^{*} / \lambda_{31, m}^{*}$ (ср. рис. $2, b$ и $c$ ). Это обусловлено влиянием на $F_{33}^{* \sigma} / F_{31}^{* \sigma}$ анизотропии пьезомодулей $d_{33}^{*} / d_{31}^{*}$. На рис. $2, g$ мы не показываем кривые ПП для композитов на основе $\mathrm{PMN}-x \mathrm{PT}$ с $x \leq 0.30$ вследствие малой разности между значениями $F_{31}^{* \sigma}(m)$ этих композитов и $F_{31}^{* \sigma}(m)$ на кривых 1,2 рис. $2, g$.

Из сравнения кривых на рис. $2, h, i$ можно установить существование интервалов объемных концентраций $m$, соответствующих большой анизотропии ПП $F_{33}^{* \xi} \gg F_{31}^{* \xi}$. Монотонное изменение упругих податливостей $s_{j j}^{* E}(m)$ и $s_{j j}^{* D}(m)$ влияет на ход кривых $F_{3 j}^{* \xi}(m)(j=1,3)$. Вследствие уменьшения $s_{j j}^{(1), E}$ KNNTL-Mn по сравнению с $s_{j j}^{(1), E} \mathrm{PMN}-0.33 \mathrm{PT}$ практически в два раза и благодаря уменьшению $\varepsilon_{33}^{(1), \sigma}$ KNNTL-Mn по сравнению с $\varepsilon_{33}^{(1), \sigma}$ $\mathrm{PMN}-0.33 \mathrm{PT}$ в 12.6 раз (табл. 1) композит на основе KNNTL-Mn обладает большим ПП $F_{33}^{* \xi}$ (кривая 5 на рис. $2, h)$. 
Таблица 2. Аналогия между концентрационными зависимостями квадратов ПП $\left(Q_{3 j}^{*}\right)^{2}$ и модифицированных ПП $F_{3 j}^{* \sigma}$ 1-3-композитов с кристаллическими стержнями в форме прямоугольного параллелепипеда

\begin{tabular}{|c|c|c|c|c|c|c|c|c|c|c|c|c|}
\hline \multirow{2}{*}{$m$} & $\begin{array}{l}\left(Q_{33}^{*}\right)^{2} \\
10^{-12} \\
\mathrm{~Pa}^{-1}\end{array}$ & $\begin{array}{l}\left(Q_{31}^{*}\right)^{2} \\
10^{-12} \\
\mathrm{~Pa}^{-1}\end{array}$ & $\begin{array}{l}F_{33}^{* \sigma} \\
10^{-12} \\
\mathrm{~Pa}^{-1}\end{array}$ & $\begin{array}{l}F_{31}^{* \sigma} \\
10^{-12} \\
\mathrm{~Pa}^{-1}\end{array}$ & $\begin{array}{l}\left(Q_{33}^{*}\right)^{2} \\
10^{-12} \\
\mathrm{~Pa}^{-1}\end{array}$ & $\begin{array}{l}\left(Q_{31}^{*}\right)^{2} \\
10^{-12} \\
\mathrm{~Pa}^{-1}\end{array}$ & $\begin{array}{l}F_{33}^{* \sigma} \\
10^{-12} \\
\mathrm{~Pa}^{-1}\end{array}$ & $\begin{array}{l}F_{31}^{* \sigma} \\
10^{-12} \\
\mathrm{~Pa}^{-1}\end{array}$ & $\begin{array}{l}\left(Q_{33}^{*}\right)^{2}, \\
10^{-12} \\
\mathrm{~Pa}^{-1}\end{array}$ & $\begin{array}{l}\left(Q_{31}^{*}\right)^{2}, \\
10^{-12} \\
\mathrm{~Pa}^{-1}\end{array}$ & $\begin{array}{l}F_{33}^{* \sigma} \\
10^{-12} \\
\mathrm{~Pa}^{-1}\end{array}$ & $\begin{array}{l}F_{31}^{* \sigma} \\
10^{-12} \\
\mathrm{~Pa}^{-1}\end{array}$ \\
\hline & \multicolumn{4}{|c|}{$\begin{array}{c}\text { Композит „кристалл } \\
\text { PMN-0.33PT-- } \\
\text { полиуретан“ }\end{array}$} & \multicolumn{4}{|c|}{$\begin{array}{c}\text { Композит „кристалл } \\
\text { PMN-0.28PT- } \\
\text { полиуретан“ }\end{array}$} & \multicolumn{4}{|c|}{$\begin{array}{c}\text { Композит „кристалл } \\
\text { KNNTL-Mn-- } \\
\text { полиуретан“ }\end{array}$} \\
\hline 0.05 & 217 & 29.9 & 81.6 & 7.80 & 92.8 & 9.65 & 33.0 & 2.47 & 167 & 22.4 & 59.3 & 5.78 \\
\hline 0.10 & 242 & 34.1 & 106 & 8.99 & 94.9 & 10.5 & 38.4 & 2.70 & 182 & 25.1 & 77.5 & 6.56 \\
\hline 0.15 & 238 & 34.5 & 113 & 9.15 & 87.5 & 10.2 & 37.7 & 2.64 & 167 & 24.0 & 77.9 & 6.28 \\
\hline 0.20 & 227 & 34.0 & 114 & 9.07 & 79.4 & 9.85 & 35.6 & $2 / 54$ & 150 & 22.4 & 73.8 & 5.88 \\
\hline 0.30 & 204 & 38.5 & 109 & 8.73 & 65.9 & 9.12 & 30.9 & 2.36 & 123 & 19.7 & 63.9 & 5.18 \\
\hline 0.50 & 165 & 29.6 & 93.7 & 8.12 & 48.4 & 8.11 & 23.7 & 2.11 & 88.5 & 16.1 & 48.4 & 4.31 \\
\hline 0.70 & 137 & 27.2 & 80.5 & 7.01 & 38.1 & 7.44 & 19.1 & 1.96 & 68.9 & 13.9 & 38.6 & 3.82 \\
\hline
\end{tabular}

Таблица 3. Сравнение эффективных параметров родственных 1-3-композитов с кристаллическими стержнями в форме прямоугольного параллелепипеда или в форме кругового цилиндра

\begin{tabular}{|c|c|c|c|c|c|c|c|c|c|c|}
\hline$m$ & $\begin{array}{c}d_{33}^{*}, \\
\mathrm{pC} / \mathrm{N}\end{array}$ & $\begin{array}{c}d_{31}^{*}, \\
\mathrm{pC} / \mathrm{N}\end{array}$ & $\lambda_{33}, m^{*}$ & $L_{33}^{*}$ & $10^{-2} \lambda_{31, m}^{*}$ & $L_{31}^{*}$ & $\begin{array}{c}F_{33}^{* \sigma}, \\
10^{-12} \mathrm{~Pa}^{-1}\end{array}$ & $\begin{array}{c}F_{31}^{* \sigma} \\
10^{-12} \mathrm{~Pa}^{-1}\end{array}$ & $\begin{array}{c}F_{33}^{* \xi}, \\
10^{10} \mathrm{~Pa}\end{array}$ & $\begin{array}{c}F_{31}^{* \xi} \\
10^{8} \mathrm{~Pa}\end{array}$ \\
\hline \multicolumn{11}{|c|}{ Композит „кристалл PN-0.33РТ-полиуретан“ } \\
\hline 0.05 & $\begin{array}{c}426 \\
(423)\end{array}$ & $\begin{array}{c}-158 \\
(-160)\end{array}$ & $\begin{array}{c}0.226 \\
(0.225)\end{array}$ & $\begin{array}{c}0.376 \\
(0.375)\end{array}$ & $\begin{array}{c}2.14 \\
(2.14)\end{array}$ & $\begin{array}{c}0.261 \\
(0.261)\end{array}$ & $\begin{array}{c}81.6 \\
(80.6)\end{array}$ & $\begin{array}{c}7.81 \\
(7.96)\end{array}$ & $\begin{array}{c}0.157 \\
(0.156)\end{array}$ & $\begin{array}{c}0.639 \\
(0.629)\end{array}$ \\
\hline 0.15 & $\begin{array}{c}1050 \\
(1050)\end{array}$ & $\begin{array}{c}-401 \\
(-403)\end{array}$ & $\begin{array}{c}0.381 \\
(0.380)\end{array}$ & $\begin{array}{c}0.477 \\
(0.476)\end{array}$ & $\begin{array}{c}3.00 \\
(2.90)\end{array}$ & $\begin{array}{c}0.264 \\
(0.264)\end{array}$ & $\begin{array}{c}113 \\
(112)\end{array}$ & $\begin{array}{c}9.15 \\
(9.30)\end{array}$ & $\begin{array}{c}0.635 \\
(0.635)\end{array}$ & $\begin{array}{c}1.11 \\
(1.01)\end{array}$ \\
\hline 0.184 & $\begin{array}{c}1220 \\
(1213)\end{array}$ & $\begin{array}{c}-469 \\
(-474)\end{array}$ & $\begin{array}{c}0.407 \\
(0.406)\end{array}$ & $\begin{array}{c}0.495 \\
(0.494)\end{array}$ & $\begin{array}{c}3.17 \\
(3.02)\end{array}$ & $\begin{array}{c}0.266 \\
(0.265)\end{array}$ & $\begin{array}{c}114 \\
(114)\end{array}$ & $\begin{array}{c}9.11 \\
(9.27)\end{array}$ & $\begin{array}{c}0.814 \\
(0.813)\end{array}$ & $\begin{array}{c}1.25 \\
(1.08)\end{array}$ \\
\hline 0.30 & $\begin{array}{c}1670 \\
(1660)\end{array}$ & $\begin{array}{c}-666 \\
(-672)\end{array}$ & $\begin{array}{c}0.462 \\
(0.462)\end{array}$ & $\begin{array}{c}0.534 \\
(0.534)\end{array}$ & $\begin{array}{c}3.70 \\
(3.53)\end{array}$ & $\begin{array}{c}0.269 \\
(0.267)\end{array}$ & $\begin{array}{c}109 \\
(108)\end{array}$ & $\begin{array}{c}8.74 \\
(8.98)\end{array}$ & $\begin{array}{l}1.45 \\
(1.45)\end{array}$ & $\begin{array}{c}1.81 \\
(1.62)\end{array}$ \\
\hline 0.50 & $\begin{array}{c}2170 \\
(2170)\end{array}$ & $\begin{array}{c}-921 \\
(-928)\end{array}$ & $\begin{array}{c}0.508 \\
(0.508)\end{array}$ & $\begin{array}{c}0.569 \\
(0.568)\end{array}$ & $\begin{array}{c}4.79 \\
(4.62)\end{array}$ & $\begin{array}{c}0.275 \\
(0.271)\end{array}$ & $\begin{array}{c}93.7 \\
(93.5)\end{array}$ & $\begin{array}{c}8.12 \\
(8.14)\end{array}$ & $\begin{array}{c}2.59 \\
(2.59)\end{array}$ & $\begin{array}{c}3.43 \\
(3.29)\end{array}$ \\
\hline \multicolumn{11}{|c|}{ Композит „кристалл KNNTL-Mn-полиуретан“ } \\
\hline 0.05 & $\begin{array}{c}147 \\
(146)\end{array}$ & $\begin{array}{c}-53.7 \\
(-55.7)\end{array}$ & $\begin{array}{c}0.191 \\
(0.190)\end{array}$ & $\begin{array}{c}0.354 \\
(0.354)\end{array}$ & $\begin{array}{c}1.67 \\
(1.61)\end{array}$ & $\begin{array}{c}0.258 \\
(0.259)\end{array}$ & $\begin{array}{c}59.3 \\
(58.6)\end{array}$ & $\begin{array}{c}5.78 \\
(5.92)\end{array}$ & $\begin{array}{c}0.132 \\
(0.132)\end{array}$ & $\begin{array}{c}0.514 \\
(0.505)\end{array}$ \\
\hline 0.125 & $\begin{array}{c}273 \\
(271)\end{array}$ & $\begin{array}{c}-102 \\
(-106)\end{array}$ & $\begin{array}{c}0.341 \\
(0.340)\end{array}$ & $\begin{array}{c}0.449 \\
(0.449)\end{array}$ & $\begin{array}{c}2.23 \\
(2.16)\end{array}$ & $\begin{array}{c}0.261 \\
(0.261)\end{array}$ & $\begin{array}{c}78.7 \\
(78.1)\end{array}$ & $\begin{array}{c}6.46 \\
(6.61)\end{array}$ & $\begin{array}{c}0.610 \\
(0.609)\end{array}$ & $\begin{array}{c}0.840 \\
(0.830)\end{array}$ \\
\hline 0.20 & $\begin{array}{c}347 \\
(346)\end{array}$ & $\begin{array}{c}-134 \\
(-138)\end{array}$ & $\begin{array}{c}0.402 \\
(0.401)\end{array}$ & $\begin{array}{c}0.492 \\
(0.491)\end{array}$ & $\begin{array}{c}2.39 \\
(2.19)\end{array}$ & $\begin{array}{c}0.262 \\
(0.261)\end{array}$ & $\begin{array}{c}73.8 \\
(73.5)\end{array}$ & $\begin{array}{c}5.89 \\
(6.04)\end{array}$ & $\begin{array}{c}1.20 \\
(1.20)\end{array}$ & $\begin{array}{c}1.07 \\
(0.962)\end{array}$ \\
\hline 0.30 & $\begin{array}{c}409 \\
(408)\end{array}$ & $\begin{array}{c}-164 \\
(-168)\end{array}$ & $\begin{array}{c}0.443 \\
(0.442)\end{array}$ & $\begin{array}{c}0.520 \\
(0.520)\end{array}$ & $\begin{array}{c}2.60 \\
(2.42)\end{array}$ & $\begin{array}{c}0.263 \\
(0.261)\end{array}$ & $\begin{array}{c}63.9 \\
(63.7)\end{array}$ & $\begin{array}{c}5.18 \\
(5.33)\end{array}$ & $\begin{array}{c}2.06 \\
(2.06)\end{array}$ & $\begin{array}{c}1.45 \\
(1.34)\end{array}$ \\
\hline 0.50 & $\begin{array}{c}477 \\
(476)\end{array}$ & $\begin{array}{c}-204 \\
(-206)\end{array}$ & $\begin{array}{c}0.480 \\
(0.479)\end{array}$ & $\begin{array}{c}0.547 \\
(0.547)\end{array}$ & $\begin{array}{c}3.31 \\
(3.14)\end{array}$ & $\begin{array}{c}0.267 \\
(0.263)\end{array}$ & $\begin{array}{c}48.4 \\
(48.3)\end{array}$ & $\begin{array}{c}4.31 \\
(4.34)\end{array}$ & $\begin{array}{c}3.84 \\
(3.84)\end{array}$ & $\begin{array}{c}2.90 \\
(2.77)\end{array}$ \\
\hline
\end{tabular}

Примечание. Эффективные параметры композитов с кристаллическими стержнями в форме кругового цилиндра приведены в скобках. $\max F_{33}^{* \sigma}(m)$ достигается при $m=0.184$ и 0.125 в композите на основе PMN-0.33PT и KNNTL-Mn соответственно независимо от формы основания стержней.

Табл. 2 иллюстрирует аналогичный характер концентрационных зависимостей $\left[Q_{3 j}^{*}(m)\right]^{2}$ и $F_{3 j}^{* \sigma}(m)$ компози- тов. По значениям $\left[Q_{33}^{*}(m)\right]^{2}$ и $F_{33}^{* \sigma}(m)$ композит на основе KNNTL-Mn занимает промежуточное положение, 
как показано в табл. 2. Значения $\max \left[Q_{33}^{*}(m)\right]^{2}$ различных композитов соответствуют меньшим объемным концентрациям $m$ по сравнению с $\max F_{33}^{* \sigma}(m)$, что связано с активным влиянием пьезокоэффициента $g_{33}^{*}(m)$ на зависимость $\left[Q_{33}^{*}(m)\right]^{2}$, особенно при $m \ll 1$. В качестве примера укажем, что согласно результатам, полученным в рамках матричного метода для композита на основе KNNTL-Mn, $\max \left[Q_{33}^{*}(m)\right]^{2}$ и $\max F_{33}^{* \sigma}(m)$ достигаются при $m=0.084$ и 0.125 соответственно.

\section{3. Сравнение результатов}

Данные табл. 3 показывают, что хорошее согласие между эффективными параметрами, рассчитанными с использованием двух методов (см. разд. 1.1), наблюдается в широком интервале $m$. Различия между численными значениями (табл. 3) обусловлены различными формами кристаллических стержней (вставки 2,3 на рис. 1) и спецификой процедур усреднения свойств (формулы (2), (5)). При этом изменение формы и электромеханических свойств стержней не приводит к заметному изменению факторов анизотропии композита

$$
\lambda_{33, m}^{*} / \lambda_{31, m}^{*} \gg 1 ; F_{33}^{* \sigma} / F_{31}^{* \sigma} \gg 1 ; F_{33}^{* \xi} / F_{31}^{* \xi} \gg 1,
$$

(табл. 3). ПП $\lambda_{33, m}^{*}$ и $L_{33}^{*} 1-3$-композитов (рис. $2, b, d$ и табл. 3) превышают аналогичные ПП пористых материалов на основе СПК типа PZT [6], что связано с более высокими значениями $d_{33}^{*}, g_{33}^{*}$ и $k_{33}^{*}$ исследованных нами композитов. Для 1-3-композита „СПК РZТ-7А-аральдит“ [45] при объемной концентрации СПК $m_{\text {cer }}=0.2$ мы получаем расчетные значения ПП $\lambda_{33, m}^{*} \approx 0.072, L_{33}^{*} \approx 0.29,\left(Q_{33}^{*}\right)^{2} \approx 16 \cdot 10^{-12} \mathrm{~Pa}^{-1}$ и $F_{33}^{* \sigma} \approx 4.6 \cdot 10^{-12} \mathrm{~Pa}^{-1}$. При этом мы исходим из экспериментальных значений [45] $d_{33}^{*} \approx 120 \mathrm{pC} / \mathrm{N}$, $\varepsilon_{33}^{* \sigma} / \varepsilon_{0} \approx 100, k_{t}^{*} \approx 0.50$ и справедливости соотношения $k_{33}^{*} \approx k_{t}^{*}$ для $1-3$-композитов в широком интервале $m[27,45]$, где $k_{t}^{*}$ 一коэффициент электромеханической связи толщиной моды. Приведенные выше значения $\lambda_{33, m}^{*}, L_{33}^{*},\left(Q_{33}^{*}\right)^{2}$ и $F_{33}^{* \sigma} 1-3$-композита на основе PZT-7А меньше соответствующих ПП, определенных в широком интервале $m$ для ряда композитов (рис. 2, $b, d, f$ и табл. 2,3). С учетом параметров 1-3-композита „кристалл РМN-0.30РТ-эпоксидная смола“ $[11] d_{33}^{*} \approx 1200 \mathrm{pC} / \mathrm{N}, \varepsilon_{33}^{* \sigma} / \varepsilon_{0} \approx 1600, k_{t}^{*} \approx 0.80$ при $m=0.56$ мы получаем $\lambda_{33, m}^{*} \approx 0.25, L_{33}^{*} \approx 0.39$, $\left(Q_{33}^{*}\right)^{2} \approx 100 \cdot 10^{-12} \mathrm{~Pa}^{-1}$ и $F_{33}^{* \sigma} \approx 39 \cdot 10^{-12} \mathrm{~Pa}^{-1}$. Примечательно, что $\left(Q_{33}^{*}\right)^{2}$ находится в интервале значений, определенных для двух композитов на основе $\mathrm{PMN}-x$ PT (табл. 2 для $x=0.33,0.28$ ), а значение $F_{33}^{* \sigma}$ приближенно равно $F_{33}^{* \sigma}$ композита „кристалл PMN0.30РТ-полиуретан“ (кривая 2 на рис. $2, f)$.

\section{Заключение}

В работе проведено исследование системы модифицированных ПП из (8)-(11) пьезоактивных 1-3-композитов на основе поляризованных вдоль [001] кристаллов PMN-xPT и KNNTL-Mn. На поведение ПП $\lambda_{3 j, m}^{*}(m) \quad$ и $L_{3 j}^{*}(m)$ в широком интервале объемных концентраций $m$ влияют коэффициенты электромеханической связи $k_{3 j}^{*}(m)$, причем монотонное поведение последних обусловливает в большинстве случаев монотонные зависимости $\lambda_{3 j, m}^{*}(m)$ (рис. 2, $\left.b, c\right)$ и $L_{3 j}^{*}(m)$ (рис. $\left.2, d, e\right)$. Немонотонные зависимости ПП $F_{3 j}^{* \sigma}(m)$ (рис. 2, $f, g$ ) объясняются важной ролью ПП $\left(Q_{3 j}^{*}\right)^{2}$ (формула (10) и табл. 2), а следовательно, пьезокоэффициентов $g_{3 j}^{*}$ согласно формулам (7). На практике легче получить композит [11] с объемной концентрацией $m>0.1$ (т.е. в области $m$, где наблюдается $\left.\max F_{3 j}^{* \sigma}(m)\right)$, чем аналогичный композит с $m<0.1$ (т.е. при концентрациях вблизи $\max \left[Q_{3 j}^{*}(m)\right]^{2}$; см. пример в конце разд. 2). Это обстоятельство выгодно отличает модифицированный ПП $F_{3 j}^{* \sigma}$ от традиционного $\Pi \Pi\left(Q_{3 j}^{*}\right)^{2}$.

В отличие от ПП $F_{3 j}^{* \sigma}$, связанных с воздействием на образец поля механических напряжений $\sigma=$ const, ПП $F_{3 j}^{* \xi}$ для случая поля деформаций $\xi=$ const не имеют экстремумов во всем интервале $m$ (рис. $2, h, i$ ). Монотонное возрастание $F_{3 j}^{* \xi}(m)$ связано с активным влиянием, согласно (11), упругих податливостей $s_{j j}^{* E}$ и $s_{j j}^{* D}$, убывающих монотонно с увеличением $m$. Исследованным 1-3-композитам присуща большая анизотропия трех видов ПП (формулы (12)) независимо от состава кристаллического компонента из табл. 1. Такие анизотропные характеристики в значительной мере обусловлены микрогеометрией композитов (рис. 1) и анизотропией свойств их кристаллических компонентов.

Сравнение эффективных параметров, рассчитанных для 1-3-композитов с помощью двух различных методов (табл. 3), позволяет сделать вывод о хорошем согласии между результатами, относящимися к четырем видам модифицированных ПП из (8)-(11). Особый интерес представляет бессвинцовый композит на основе KNNTL-Mn, способный благодаря большим значениям ряда ПП (рис. $2, b, d, f, g, h, i$ ) конкурировать с композитами на основе кристаллов PMN $-x$ PТ или СПК типа PZT. Исследованные 1-3-композиты целесообразно применять как пьезоэлектрические преобразователи, сенсоры и элементы энергосберегающих устройств, работающих при $\sigma=$ const или $\xi=$ const. Такие устройства рассмотрены, например, в работе [38] (накопление и преобразование энергии при воздействии внешней силы) и патенте [46] (пьезоэлектрический элемент, на который действует пружина при условии $\xi=$ const).

\section{Благодарности}

Авторы выражают благодарность РФФИ за эту поддержку. Авторы благодарны проф. А.Е. Паничу (ЮФУ) и проф. C.R. Bowen (University of Bath, Соединенное Королевство) за постоянный интерес к тематике исследований и полезные дискуссии по вопросам эффективности 
современных пьезоэлектрических материалов. Авторы выражают особую благодарность д.т.н. И.А. Паринову (ЮФУ) и рецензенту за внимательное изучение рукописи статьи и полезные замечания.

\section{Финансирование работы}

Исследование выполнено при финансовой поддержке РФФИ в рамках научного проекта № 20-38-90163.

\section{Конфликт интересов}

Авторы заявляют, что у них нет конфликта интересов.

\section{Список литературы}

[1] S. Priya. IEEE Trans. Ultrason., Ferroelec., a. Freq. Contr., 57 (12), 2010 (2010). DOI: 10.1109/TUFFC.2010.1734

[2] G.M. Sessler, J. Hillenbrand. Appl. Phys. Lett., 103 (12), 122904 (2013). http://dx.doi.org/10.1063/1.4821647

[3] C.R. Bowen, V.Yu. Topolov, H.A. Kim. Modern Piezoelectric Energy Harvesting (Springer International Publishing Switzerland, Cham, 2016)

[4] J.I. Roscow, R.W.C. Lewis, J. Taylor, C.R. Bowen. Acta Mater., 128, 207 (2017). DOI: 10.1016/j.actamat.2017.02.029

[5] K. Uchino. Energy Technol., 6 (5), 829 (2018). DOI: $10.1002 /$ ente. 201700785

[6] J.I. Roscow, H. Pearce, H. Khanbareh, S. Kar-Narayan, C.R. Bowen. Eur. Phys. J. Special Topics, 228 (7), 1537 (2019). https://doi.org/10.1140/epjst/e2019-800143-7

[7] T. Rödig, A. Schönecker, G. Gerlach. J. Am. Ceram. Soc., 93 (4), 901 (2010). https://doi.org/10.1111/j.15512916.2010.03702.x

[8] Пьезоэлектрическое приборостроение, под ред. А.В. Гориша. Т. 1. Физика сегнетоэлектрической керамики (Радиотехника, М., 1999)

[9] L. Jiang, R. Chen, J. Xing, G. Lu, R. Li, Y. Jiang, K. Shung, J. Zhu, Q. Zhou. J. Appl. Phys., 125 (21), 214501 (2019). https://doi.org/10.1063/1.5088171

[10] Q. Ke, W.H. Liew, H. Tao, J. Wu, K. Yao. IEEE Trans. Ultrason., Ferroelec., a. Freq. Contr., 66 (8), 1395 (2019). DOI: 10.1109/TUFFC.2019.2914464

[11] F. Wang, C. He, Y. Tang, X. Zhao, H. Luo. Mater. Chem. Phys., 105 (2-3), 273 (2007). DOI: 10.1016/j.matchemphys.2007.04.060

[12] D. Zhou, K.H. Lam, Y. Chen, Q. Zhang, Y.C. Chiu, H. Luo, J. Dai, H.L.W. Chan. Sens. Actuators A-Phys., 182 (1), 95 (2012).

[13] Z. Yang, D. Zeng, H. Wang, C. Zhao, J. Tan. Smart Mater. Struct., 24 (7), 075029 (2015). DOI: $10.1088 / 0964-1726 / 24 / 7 / 075029$

[14] Б.Е. Победря. Механика композиционных материалов (Изд-во Моск. ун-та, М., 1984)

[15] Б.Е. Победря. Механика композитных материалов, 32 (6), 729 (1996). [B.E. Pobedrya. Mech. Compos. Mater., 32 (6), 504 (1996). DOI: 10.1007/BF02280632]

[16] M.L. Dunn. J. Appl. Phys., 73 (10), 5131 (1993). DOI: $10.1063 / 1.353787$
[17] V.M. Levin, M.I. Rakovskaja, W.S. Kreher. Internat. J. Solids Struct., 36 (18), 2683 (1999). DOI: $10.1016 /$ S0020-7683(98)00131-0

[18] N. Fakri, L. Azrar, L. El Bakkali. Internat. J. Solids Struct., 40 (2), 361 (2003). DOI: 10.1016/S0020-7683(02)00524-3

[19] С.К. Канаун, В.М. Левин. Метод эффективного поля в механике композитных материалов (Изд-во Петрозав. ун-та, Петрозавод., 1993)

[20] S. Kanaun, V. Levin. In: Effective Properties of Heterogeneous Materials, ed. by M. Kachanov, I. Sevostianov (Springer, Dordrecht, 2013), p. 199.

[21] Л.П. Хорошун, Б.П. Маслов, П.В. Лещенко. Прогнозирование эффективных свойств пьезоактивных композитных материалов (Наук. думка, Киев, 1989)

[22] А.А. Паньков. Статистическая механика пьезокомпозитов (Изд-во Перм. гос. техн. ун-та, Пермь, 2009)

[23] A.L. Kalamkarov, K.S. Challagulla. In: Effective Properties of Heterogeneous Materials, ed. by M. Kachanov, I. Sevostianov (Springer, Dordrecht, 2013), p. 283.

[24] F. Levassort, M. Lethiecq, C. Millar, L. Pourcelot. IEEE Trans. Ultrason., Ferroelec., Freq. Contr., 45 (5), 1497 (1998). DOI: $10.1109 / 58.738289$

[25] V.Yu. Topolov, A.V. Krivoruchko, P. Bisegna, C.R. Bowen. Ferroelectrics, 376, 140 (2008). DOI: $10.1080 / 00150190802440997$

[26] V.Yu. Topolov, P. Bisegna, A.V. Krivoruchko. J. Phys. D: Appl. Phys., 41 (3), 035406 (2008). DOI: $10.1088 / 0022-3727 / 41 / 3 / 035406$

[27] V.Yu. Topolov, C.R. Bowen, P. Bisegna. Piezo-Active Composites. Microgeometry -Sensitivity Relations (Springer International Publishing Switzerland, Cham, 2018)

[28] Ю.В. Соколкин, А.А. Паньков. Электроупругость пьезокомпозитов с нерегулярными структурами (Физматлит, М., 2003)

[29] S.V. Bezus, V.Yu. Topolov, C.R. Bowen. J. Phys. D: Appl. Phys., 39 (9), 1919 (2006). DOI: 10.1088/0022-3727/39/9/029

[30] R. Zhang, B. Jiang, W. Cao. J. Appl. Phys., 90 (7), 3471 (2001). https://doi.org/10.1063/1.1390494

[31] M.L. Dunn, M. Taya. Internat. J. Solids Struct., 30 (2), 161 (1993). https://doi.org/10.1016/0020-7683(93)90058-F

[32] А.В. Турик. ФТT, 12 (3), 892 (1970). [A.V. Turik. Soviet Phys.-Solid State, 12 (3), 688 (1970).]

[33] W.A. Smith. IEEE Trans. Ultrason., Ferroelec., Freq. Contr., 40 (1), 41 (1993). DOI: 10.1109/58.184997

[34] O. Sigmund, S. Torquato, I.A. Aksay, J. Mater. Res., 13 (4), 1038 (1998). DOI: 10.1557/JMR.1998.0145

[35] J.H. Huang, S. Yu. Compos. Engin., 4 (11), 1169 (1994). https://doi.org/10.1016/0961-9526(95)91290-W

[36] L. Li, N.R. Sottos. J. Appl. Phys., 77 (9), 4595 (1995). https://doi.org/10.1063/1.359424

[37] J. Bennett, G. Hayward. IEEE Trans. Ultrason., Ferroelec., a. Freq. Contr., 44 (3), 565 (1997). DOI: 10.1109/58.658308

[38] D.B. Deutz, J.-A. Pascoe, B. Schelen, S. van der Zwaag, D.M. de Leeuw, P. Groen. Mater. Horiz., 5 (3), 444 (2018). https://doi.org/10.1039/C8MH00097B

[39] R. Zhang, W. Jiang, B. Jiang, W. Cao. Fundamental Physics of Ferroelectrics, ed. by R.E. Cohen (American Institute of Physics, Melville, 2002), p. 188.

[40] S. Zhang, J. Luo, W. Hackenberger, T.R. Shrout. J. Appl. Phys., 104 (6), 064106 (2008). DOI: 10.1063/1.2978333

[41] G. Liu, W. Jiang, J. Zhu, W. Cao. Appl. Phys. Lett., 99 (16), 162901. https://doi.org/10.1063/1.3652703 
[42] X. Huo, R. Zhang, L. Zheng, S. Zhang, R. Wang, J. Wang, S. Sang, B. Yang, W. Cao. J. Am. Ceram. Soc., 98 (6), 1829 (2015). DOI: $10.1111 /$ jace. 13540

[43] L.V. Gibiansky, S. Torquato. J. Mech. Phys. Sol., 45 (5), 689 (1997). DOI: 10.1016/S0022-5096(96)00106-8

[44] B. Noheda. Curr. Opin. Solid State Mater. Sci., 6 (1), 27 (2002). https://doi.org/10.1016/S1359-0286(02)00015-3

[45] H.L.W. Chan, J. Unsworth. IEEE Trans. Ultrason., Ferroelec., a. Freq. Contr., 36 (4), 434 (1989). DOI: 10.1109/58.31780

[46] D.L. Churchill, S.W. Arms. Piezoelectric Composite with Tapered Beam, Patent US 7880370 B2, 01.02.2011 